THE ASTROPHYSICAL JoURNAL, 510:846-853, 1999 January 10

(C) 1999. The American Astronomical Society. All rights reserved. Printed in U.S.A.

\title{
GRAVITATIONAL RADIATION LIMIT ON THE SPIN OF YOUNG NEUTRON STARS
}

\author{
NILS ANDERSSON \\ Institut für Astronomie und Astrophysik, Universität Tübingen, D-72076 Tübingen, Germany; and Department of Mathematics, \\ University of Southampton, Southampton, UK \\ Kostas KoKKOTAS \\ Department of Physics, Aristotle University of Thessaloniki, Thessaloniki 54006, Greece \\ AND \\ BERNARD F. SCHUTZ \\ Max Planck Institute for Gravitational Physics, Albert Einstein Institute, D-14473 Potsdam, Germany \\ Received 1998 May 18; accepted 1998 August 18
}

\begin{abstract}
A newly discovered instability in rotating neutron stars, driven by gravitational radiation reaction acting on the stars' $r$-modes, is shown here to set an upper limit on the spin rate of young neutron stars. We calculate the timescales for the growth of linear perturbations due to gravitational radiation reaction, and for dissipation by shear and bulk viscosity, working to second order in a slow-rotation expansion within a Newtonian polytropic stellar model. The results are very temperature-sensitive: in hot neutron stars $\left(T>10^{9} \mathrm{~K}\right)$, the lowest-order $r$-modes are unstable, while in colder stars they are damped by viscosity. These calculations have a number of interesting astrophysical implications. First, the $r$-mode instability will spin down a newly born neutron star to a period close to the initial period inferred for the Crab pulsar, probably between 10 and $20 \mathrm{~ms}$. Second, as an initially rapidly rotating star spins down, an energy equivalent to roughly $1 \%$ of a solar mass is radiated as gravitational waves, which makes the process an interesting source for detectable gravitational waves. Third, the $r$-mode instability rules out the scenario in which millisecond pulsars are formed by accretion-induced collapse of a white dwarf; the new star would be hot enough to spin down to much slower rates. Stars with periods less than perhaps 10 ms must have been formed by spin-up through accretion in binary systems, where they remain colder than the Eddington temperature of about $10^{8} \mathrm{~K}$. More accurate calculations will be required to define the limiting spin period more reliably, and we discuss the importance of the major uncertainties in the stellar models, in the initial conditions after collapse, and in the physics of cooling, superfluidity, and the equation of state.
\end{abstract}

Subject headings: gravitation - stars: neutron — stars: oscillations - stars: rotation

\section{INTRODUCTION}

One of the puzzles of pulsar astrophysics is that neutron stars seem to be formed with rather small initial spin rates. The best-studied young pulsar is the Crab (PSR 0531+21), whose initial period is estimated to have been about $19 \mathrm{~ms}$ (Glendenning 1996). Even the recently discovered young 16 ms X-ray pulsar in the supernova remnant N157B (Marshall et al. 1998) probably had an initial period no shorter than 6-9 ms (assuming a braking index typical of young pulsars). This contrasts with the shortest known period of a recycled pulsar of $1.6 \mathrm{~ms}$, and with the theoretical lower limit on the period of about 0.5 to $2 \mathrm{~ms}$, depending on the equation of state (Friedman, Parker, \& Ipser 1989). If angular momentum is conserved in the collapse event that forms the neutron star, it would not be surprising to find young pulsars spinning at or close to the theoretical maximum.

In this paper, we offer an explanation for the observed slow initial rotation rates by showing that, if a new neutron star has a period shorter than some critical value $P_{f}$, then within a year its period will increase to $P_{f}$. During this spin-down period it will lose angular momentum to gravitational radiation through the growth of a radiation-driven nonaxisymmetric instability associated with the $r$-modes. The final rotation period $P_{f}$ depends on details of cooling rates, viscosity, and perhaps on initial conditions following collapse. For the simple models of neutron stars that we compute in this paper, this period is about $20 \mathrm{~ms}$, consistent with the estimated initial spin of the Crab pulsar, given the various uncertainties in our calculation (see below). These uncertainties are, in fact, probably sufficient to include the presumed initial period of the recently discovered pulsar in N157B. We discuss this further later in the paper. The instability operates mainly in hot neutron stars $\left(T>10^{9} \mathrm{~K}\right)$; neutron stars that are spun up in accreting systems do not reach this temperature, and we do not expect the instability to put a strong constraint on their speeds (see Andersson, Kokkotas, \& Stergioulas 1998 for a discussion of the potential relevance of the instability in such systems).

An alternative explanation for the slow initial rotation of pulsars was recently suggested by Spruit \& Phinney (1998). They argue that magnetic locking between the core and the envelope of the progenitor star prevents the core from spinning rapidly. The core would, in fact, rotate far too slowly to lead (by conservation of angular momentum) to young neutron stars spinning as fast as they actually do. Spruit \& Phinney (1998) propose that the neutron-star spin is due to the birth kicks that also produce the large linear velocities observed for pulsars. This model has implications for the evolution of the angular momentum of the degenerate cores of presupernova giants, and it also reduces expectations that gravitational collapse may be followed by a strong burst of gravitational radiation. However, even if the suggested mechanism is operating and explains the slow initial rotation of most pulsars, some pulsars should be born spinning rapidly. In the proposed model, kicks that lead to 
velocities larger than $500 \mathrm{~km} \mathrm{~s}^{-1}$ can also produce rotation at periods shorter than $10 \mathrm{~ms}$. Furthermore, the $16 \mathrm{~ms}$ pulsar in N157B is clear evidence that there is a class of pulsars that form spinning rapidly (with periods shorter than say 10-20 ms). For these pulsars, the $r$-mode instability should be relevant, and it is the rotation rate of such young short-period pulsars that we discuss below.

In addition to explaining the slow rotation of young pulsars, the $r$-mode instability has another important consequence. Millisecond pulsars (with periods shorter than $P_{f}$ ) can only reach their rotation speeds by being spun up again after they cool down. The alternative model, in which these stars are formed by accretion-induced collapse of a white dwarf, is inconsistent with this instability; the collapse would form a star hot enough to spin down within a year. So the $r$-mode instability gives further weight in support of the standard picture that millisecond pulsars are a distinct class of recycled pulsars that acquired their angular momentum through accretion in binary systems.

The $r$-mode instability is a member of the class of gravitational-radiation-driven instabilities called CFS instabilities (Thorne 1987, p. 330), after Chandrasekhar (1970), who discovered them, and Friedman \& Schutz (1978), who showed that they are generic in rotating neutron stars. The criterion for and mechanism of this instability are simple: if the pattern speed of the mode is forward-going as seen from far away, but backward-going with respect to the rotation of the star, then when the mode radiates away angular momentum, the star can find a rotation state of lower angular momentum and lower energy. This allows the mode amplitude to grow. For a mode with angular pattern speed $\sigma$ in an ideal inviscid uniformly rotating star of angular velocity $\Omega$, this means that there will be an instability if

$$
0<\sigma<\Omega .
$$

The mechanism is much the same as that which drives the Kelvin-Helmholz instability, raising waves in the sea (Schutz 1983). Viscosity, however, acts on the mode in a different way, and if large enough will damp out the instability.

Many families of oscillation modes of neutron stars contain members that, for a sufficiently high stellar rotation rate, satisfy the instability criterion (eq. [1]). Until recently, it was believed that viscosity in neutron stars stabilized all of them except in very limited regimes (Lindblom 1995). But the picture changed when Andersson (1998) pointed out that a hitherto unstudied family of modes, the $r$-modes, which are generalizations of the Rossby waves familiar to oceanographers, should also exhibit the instability. He noticed that, uniquely among the families of neutron-star normal modes, the $r$-modes satisfy the instability criterion (eq. [1]) for any stellar rotation rate, no matter how small. He attempted a preliminary estimate of the growth rate of these modes, which showed that it might be interesting and was worth further investigation. Friedman \& Morsink (1998) then showed that the instability is generic: every $r$-mode is in principle unstable in every rotating star, in the absence of viscosity.

To show that the instability is interesting for neutron-star evolution, however, it is necessary both to accurately calculate the growth rate of the mode due to gravitational radiation reaction and to estimate the damping caused by viscosity. In this paper, and in independent work by Lind- blom et al. (1998), it is shown that the $r$-mode instability is surprisingly effective. In a rapidly rotating newly formed star, the mode grows rapidly and remains unstable during the time it takes the star to cool to about $10^{9} \mathrm{~K}$, about $1 \mathrm{yr}$ using conventional cooling rates. During this time, the gravitational radiation emitted by the mode will remove most of the star's initial angular momentum, until it spins down to roughly the period $P_{f}$, where viscosity dominates gravitational radiation for any temperature.

The $r$-modes are unusual in several respects. They are primarily perturbations of the velocity field in the star, with little disturbance in the star's density. In a nonrotating star, they are neutral rotational motions. In a rotating star, Coriolis effects provide a weak restoring force that gives them genuine dynamics. The gravitational radiation that $r$-modes emit comes primarily from their time-dependent mass currents. This is the gravitational analogue of magnetic multipole radiation. We believe that this instability is unique among expected astrophysical sources of gravitational radiation in radiating primarily by gravitomagnetic effects, the same sector of relativistic gravitation theory that is responsible for the Lense-Thirring effect near rotating bodies and the Penrose process near spinning black holes (see, e.g., Misner, Thorne, \& Wheeler 1973). The present paper and Lindblom et al. (1998) give the first calculations of the strength of gravitomagnetic radiation from stellar perturbations.

Like magnetic multipole radiation, gravitomagnetic radiation of any multipole is $(v / c)^{2}$ weaker than radiation from the corresponding mass (electric) multipole of the same amplitude, where $v$ is the typical velocity associated with the perturbation. Despite this, the quadrupole $(l=2) r$-mode is more strongly unstable to gravitational radiation than any other mode so far examined in neutron stars, such as, for example, the density-dominated $f$-mode (Lindblom 1995). The reason for this is that the $r$-modes are unstable in any inviscid star, while the quadrupole $f$-mode is never more than marginally unstable in the most rapidly rotating stars, and the higher-order $f$-modes do not radiate any more strongly than the $r$-mode.

Our initial calculation of the strength and importance of the $r$-mode instability necessarily involves approximations. The neutron stars are treated as Newtonian polytropes, the modes are treated in a slow-rotation approximation, damping effects due to viscosity are assumed to be uniform throughout the star, and the stars are assumed to be uniformly rotating. An important difference between our calculations and the independent calculations of Lindblom et al. (1998), which were completed somewhat earlier, is that we carry our calculations to a sufficiently high order in the star's angular velocity $\Omega$, in order to fully calculate the small density perturbation associated with the mass motions of the $r$-modes. This is important for correctly estimating the effects of bulk viscosity, which dominates the evolution of the spin of the star at high temperatures. Reassuringly, despite this and other smaller differences, the conclusions of the two studies are broadly the same. It seems unlikely to us that when more accurate calculations are performed the results will be markedly different qualitatively, although there could be quantitative differences in the limiting period and in details of the spin-down behavior.

The evolution of the spin of the neutron star and the precise value of the limiting period $P_{f}$ depend also on the physics of neutron-star viscosity, cooling, and stratification. 
We have adopted the best available models of cooling and viscosity (see references in the body of this paper), but there are important uncertainties, particularly concerning the onset of superfluidity. We have ignored stratification (differentiation of the crust from the interior, for example, or differential rotation of the interior). These uncertainties can also have a significant effect on the value of $P_{f}$. We hope that the fact that $P_{f}$ is in principle an observable, and the possibility that gravitational radiation from the spin-down may eventually also be directly observed, will encourage further work on the critical physics issues here.

In a separate paper (Owen et al. 1998), the implications of this instability for gravitational radiation are examined. That paper contains a first attempt at developing a timedependent model of the evolution of the instability, including the nonlinear phase in which it is radiating away most of the angular momentum of the star in a steady way. The conclusions reached are that (1) the radiation emitted by a young neutron star in the Virgo cluster can be directly detected by the advanced versions of the LIGO (Abramovici et al. 1992) and VIRGO (Bradaschia et al. 1990) gravitational-wave detectors now under construction, and (2) the radiation emitted by all the neutron stars created since the beginning of star formation should form a random background of gravitational radiation that may be detectable by the same instruments. Such detections could come as early as 2005 . We will then have a direct means of investigating the physics that affects the evolution of this instability in neutron stars.

The plan of this paper is as follows. In $\S 2$ we describe the perturbation formalism and the nature of the $r$-modes, and we calculate the effects of gravitational radiation, bulk viscosity, and shear viscosity on them. The fundamental limiting period $P_{f}$ is derived in this section. Section 3 draws our main astrophysical conclusions and discusses the chief physics uncertainties that affect the computation of the limiting period $P_{f}$. We examine various possible spin-down scenarios, and identify the key uncertainties that can be addressed in future studies.

\section{ESTIMATED TIMESCALES}

To investigate the astrophysical relevance of the $r$-mode instability, we have calculated the mode frequencies and eigenfunctions from a Newtonian slow-rotation expansion accurate to order $\Omega^{2}$. Our mode calculation essentially repeats that of Saio (1982), and in order to keep the presentation compact we use his notation here. All original equations can be found in his paper. For the modes that are most strongly affected by the instability (those corresponding to $l=m$ ), the calculation leads to a displacement vector

$$
\frac{\bar{\xi}}{a}=\left(S_{l+1 l} Y_{l+1 l}, K_{l l} \sin ^{-1} \theta \partial_{\phi} Y_{l l},-K_{l l} \partial_{\theta} Y_{l l}\right) e^{i \omega t},
$$

where $Y_{l m}$ are the standard spherical harmonics, and $K_{l l}$ and $S_{l+1 l}$ are eigenfunctions of order 1 and $\Omega^{2}$, respectively. The variable $a$ is related to the radial distance $r$ through $r=a(1+\epsilon)$, where $\epsilon \sim \Omega^{2}$ is the rotationally induced deformation of the star. We incorporate these deformations by using a polytropic model created according to the procedure of Chandrasekhar \& Lebovitz (1962). Saio's equations also determine an eigenfunction $\zeta_{l+1 l}$ that is related to the Eulerian perturbation of the pressure through $\delta p=$ $\rho g a \zeta_{l+1 l} Y_{l+1 l}$, where $g=M(a) / a^{2}$ is the local acceleration of gravity and $M(a)$ is the mass inside radius $a$.

In our calculation of the $r$-mode eigenfunctions, we make use of the Cowling approximation, i.e., we neglect the perturbations of the gravitational potential. This is not strictly necessary, but the resulting equations are simpler, and it has been shown that the error associated with this approximation is very small for the $r$-modes (Saio 1982; Provost, Berthomieu, \& Rocca 1981). This is natural, since the density perturbation associated with each $r$-mode is small.

The character of the $r$-modes depends crucially on the stratification in the star. In consequence, calculations for convective (Schwarzschild discriminant $A>0$ ) and radiative $(A<0)$ models lead to rather different spectra (Provost et al. 1981; Saio 1982). In general, the $r$-modes are degenerate at frequency

$$
\omega=-m \Omega \frac{(l-1)(l+2)}{l(l+1)},
$$

measured in the inertial frame, to leading order (Papaloizou $\&$ Pringle 1978). At higher orders in $\Omega$, the degeneracy of the $r$-modes is then broken, and modes whose eigenfunctions behave differently on the radial coordinate $r$ (e.g., have a different number of nodes) become distinct (Saio 1982). Thus, an acceptable overall solution to Saio's equations can be found only for a discrete set of eigenvalues that correspond to small (order $\Omega^{3}$ ) corrections to equation (3). The barotropic perturbations $(A=0)$ that we consider in the present paper are somewhat different. First, we find no $r$-mode solutions for $l \neq m$. This is in accord with the result of Provost et al. (1981). For $l=m$, we find a single mode solution to Saio's equations. The particulars of this solution are easily understood. In the case of barotropic perturbations, Saio's equations simplify considerably and can be written as

$$
a \frac{d S_{l+1 l}}{d a}=-\left[l+4-\frac{M(a)}{a c^{2}}\right] S_{l+1 l}-\left[h+\frac{M(a)}{a c^{2}}\right] \zeta_{l+1 l},
$$

where $c$ is the sound speed, and the variable $h$ depends on the rotationally induced deformation of the star (see Saio 1982 for the definition of $h$ ). We also have

$$
a \frac{d \zeta_{l+1 l}}{d a}=\left[l+2-\frac{4 \pi \rho a^{3}}{M(a)}\right] \zeta_{l+1 l} .
$$

A mode solution to these equations is regular at the center of the star and satisfies the condition that the Lagrangian variation in the pressure vanishes at the surface. Given such a solution, the toroidal component of the displacement vector follows from the algebraic relation

$$
\zeta_{l+1 l}=-\frac{2 i}{\sqrt{2 l+3}} \frac{l}{l+1}(\omega+l \Omega) \Omega \sqrt{\frac{M}{R^{3}}} \frac{a^{3}}{M(a)} K_{l l} .
$$

The barotropic case is peculiar, because the equation for $\zeta_{l+1 l}$ is completely decoupled. Furthermore, from equations (5) and (6), one can easily deduce that the equation allows a solution $K_{l l}=a^{l-1}$. To find an $r$-mode for the barotropic case, we combine this solution with the numerical solution to equation (4). We then find a single mode solution (that satisfies the boundary conditions) for a dimensionless eigen- 
value $\omega_{2}$, where

$$
\omega=-\frac{\Omega}{l+1}\left[(l-1)(l+2)-2 \omega_{2} \frac{R^{3} \Omega^{2}}{M}\right] .
$$

For the identified solution, we find that $S_{l+1 l} \approx \zeta_{l+11}$. In the following discussion we will only consider this specific solution.

At this point, it is legitimate to worry that the peculiarities of the barotropic case are such that the properties of the mode we consider may be radically different in the general case (which could affect the main conclusions below). It is clearly important to ensure that this is not the case. Hence, we have also done calculations for nonbarotropic models. We then find that the specific $r$-mode we consider in this paper (for which $K_{l l} \sim a^{l-1}$ ) is hardly affected at all by changes in the adiabatic index. This is somewhat surprising, since the other $r$-modes that exist in the nonbarotropic case are strongly dependent on the adiabatic index (cf. Saio 1982), but it gives us confidence that our conclusions regarding the instability of the specific mode we consider will also be relevant in the general case.

It should be noted that the $r$-mode frequency always has different signs in the inertial and the rotating frames. That is, although the modes appear retrograde in the rotating system, an inertial observer would view them as prograde. To leading order, the pattern speed of the modes is

$$
\sigma=\Omega \frac{(l-1)(l+2)}{l(l+1)} .
$$

Since $0<\sigma<\Omega$ for all $l \geq 2$, the $r$-modes are destabilized by the standard CFS mechanism and are unstable because of the emission of gravitational waves (cf. eq. [15]).

We have done a series of calculations for barotropic perturbations of a polytropic stellar model $p=\kappa \rho^{\Gamma}$ with $\Gamma=2$. The numerical results listed in Table 1 were obtained for a model with $R=12.47 \mathrm{~km}$ and $M=2.21 \mathrm{~km}=1.5 M_{\odot}$. The absolute upper limit of rotation is set by the Kepler frequency at which mass shedding at the equator occurs. This frequency is well approximated by $\Omega_{\mathrm{K}} \approx 0.67(\pi G \bar{\rho})^{1 / 2}$ (where $\bar{\rho}$ is the average density of the star) for many equations of state. For our chosen stellar model, we have $\Omega_{\mathrm{K}} \approx$ $5.6 \times 10^{3} \mathrm{~s}^{-1}$, i.e., a minimum period of $P_{\mathrm{K}}=2 \pi / \Omega_{\mathrm{K}} \approx$ $1.12 \mathrm{~ms}$.

In order to estimate the growth timescale for the $r$-mode instability, we need to consider the dissipation due to gravitational waves as well as the effects of bulk and shear viscosity. To do this, we use approximations that have been used to study unstable $f$-modes (Ipser \& Lindblom 1991).

\section{TABLE 1}

Estimated Timescales for the Various Dissipation Mechanisms RELEVANT TO THE $r$-MODE INSTABILITY

\begin{tabular}{ccccccc}
\hline \hline$l$ & $m$ & $\begin{array}{c}\tau_{\mathrm{gw}} \\
(\mathrm{s})\end{array}$ & $p_{\mathrm{gw}}$ & $\begin{array}{c}\tau_{\mathrm{bv}} \\
(\mathrm{s})\end{array}$ & $p_{\mathrm{bv}}$ & \multicolumn{1}{c}{$\begin{array}{c}\tau_{\mathrm{sv}} \\
(\mathrm{s})\end{array}$} \\
\hline $2 \ldots \ldots$ & 2 & 20.83 & 5.93 & $9.3 \times 10^{10}$ & 1.77 & $2.25 \times 10^{8}$ \\
$3 \ldots \ldots$ & 3 & 316.1 & 7.98 & $1.89 \times 10^{10}$ & 1.83 & $3.53 \times 10^{7}$ \\
\hline
\end{tabular}

NoTe.-The estimates were obtained by fitting $t=\tau(P / 1 \mathrm{~ms})^{p} \mathrm{~s}$, where $t$ is the $e$-folding time associated with each mechanism and $P$ is the rotation period of the star, to numerically obtained data. The gravitational-wave results are indicated by the index "gw;" the bulk viscosity is " bv," and the shear viscosity is "sv" (notably independent of $P$ ). The timescale data were obtained for a star at $10^{9} \mathrm{~K}$.
Specifically, we assume that the timescale $\left(t_{d}\right)$ associated with any dissipation mechanism is estimated by

$$
\frac{d E}{d t}=-\frac{2 E}{t_{d}} .
$$

Here, $E$ is the energy of the mode measured in the rotating frame. That is, we have (to leading order)

$$
E=\frac{1}{2} \int \rho|\dot{\xi}|^{2} d V=\frac{l(l+1)}{2}(\omega+l \Omega)^{2} \int \rho a^{4}\left|K_{l l}\right|^{2} d a .
$$

The dissipation due to gravitational waves can be calculated from the standard multipole formula (Thorne 1980; Ipser \& Lindblom 1991)

$$
\left.\frac{d E}{d t}\right|_{\mathrm{gw}}=-(\omega+m \Omega) \sum_{l=2}^{\infty} N_{l} \omega^{2 l+1}\left(\left|\delta D_{l m}\right|^{2}+\left|\delta J_{l m}\right|^{2}\right),
$$

where

$$
N_{l}=4 \pi \frac{(l+1)(l+2)}{l(l-1)[(2 l+1) ! !]^{2}} .
$$

The mass multipoles follow from

$$
\delta D_{l m}=\int \delta \rho a^{l} Y_{l m}^{*} d V
$$

and the current multipoles are given by

$$
\delta J_{l m}=2 \sqrt{\frac{l}{l+1}} \int a^{l}(\rho \delta \bar{v}+\delta \rho \bar{v}) \bar{Y}_{l m}^{B *} d V,
$$

where $\bar{Y}_{l m}^{B}$ are the magnetic multipoles defined by Thorne (1980). Since we are only interested in the leading-order contribution for the $l=m$ modes here, the expression for the gravitational-wave luminosity simplifies considerably. Recalling that the Eulerian variation of the density is second order $\left(\delta \rho \sim \Omega^{2}\right)$, and that $\delta \bar{v}=\dot{\bar{\xi}} \sim \Omega$, we see that the dominant contribution for $l=m$ modes will come from the first term in equation (14). Hence, we only need to evaluate

$$
\left.\frac{d E}{d t}\right|_{\mathrm{gw}}=-4 l^{2} N_{l}(\omega+l \Omega)^{3} \omega^{2 l+1}\left|\int_{0}^{R} \rho a^{l+3} K_{l l} d a\right|^{2} .
$$

It should be noted that the right-hand side of this equation is positive for the modes we are discussing. This indicates that the emission of gravitational radiation causes a growth in the mode energy in the rotating frame, despite the decrease in the inertial-frame energy. At first sight this may seem puzzling, but it can be understood from the relation between the two energies,

$$
E_{\text {rot }}=E_{\text {inertial }}-\Omega J \text {. }
$$

From this it is clear that $E_{\text {rot }}$ may increase if both $E_{\text {inertial }}$ and $J$ decrease (Friedman \& Schutz 1978).

The timescale at which the mode instability grows because of gravitational-wave emission is relevant, but it does not in itself establish that we are dealing with an astrophysical effect. It is well known that viscosity tends to 
suppress a gravitational-wave instability (Cutler \& Lindblom 1987). For the instability to be relevant, it must grow sufficiently fast that it is not completely damped out by viscosity. For neutron stars, two kinds of viscosity-bulk and shear-are normally considered. The dissipation due to bulk viscosity follows from

$$
\left.\frac{d E}{d t}\right|_{\mathrm{bv}}=-\int \zeta|\delta \sigma|^{2} d V
$$

where

$$
\delta \sigma=-i(\omega+m \Omega) \frac{\Delta p}{\Gamma p} .
$$

Here $\Delta p$ is the Lagrangian variation in the pressure, which can be deduced from (in the notation of Saio 1982)

$$
\Delta p=\delta p+\frac{d p}{d a} \xi^{a}=\rho g a \sum_{l m}\left(\zeta_{l m}-S_{l m}\right) Y_{l}^{m},
$$

since $d p / d a=-\rho g$ for a Newtonian model. The relevant viscosity coefficient is

$$
\begin{aligned}
\zeta=6 \times 10^{25}\left(\frac{\rho}{10^{15} \mathrm{~g} \mathrm{~cm}^{-3}}\right)^{2}\left(\frac{\omega+m \Omega}{1 \mathrm{~Hz}}\right)^{-2} \\
\times\left(\frac{T}{10^{9} \mathrm{~K}}\right)^{6} \mathrm{~g} \mathrm{~cm}^{-1} \mathrm{~s}^{-1} .
\end{aligned}
$$

After solving the angular integrals, we get (for $l=m$ )

$$
\left|\frac{d E}{d t}\right|_{\mathrm{bv}}=-\int_{0}^{R} \zeta\left(\frac{\rho g a^{2}}{\Gamma p}\right)^{2}\left(\zeta_{l+1 l}-S_{l+1 l}\right)^{2} d a .
$$

The dissipation rate due to shear viscosity follows from

$$
\left.\frac{d E}{d t}\right|_{\mathbf{s v}}=-2 \int \eta \delta \sigma^{a b} \delta \sigma_{a b}^{*} d V,
$$

where

$$
\delta \sigma_{a b}=\frac{i(\omega+m \Omega)}{2}\left(\nabla_{a} \xi_{b}+\nabla_{b} \xi_{a}-2 g_{a b} \nabla_{c} \xi^{c}\right) .
$$

This leads to an integral

$$
\begin{aligned}
\left.\frac{d E}{d t}\right|_{\mathrm{sv}}= & -(\omega+l \Omega)^{2} l(l+1) \\
& \times\left\{\int_{0}^{R} \eta a^{2}\left|a \partial_{a} K_{l l}\right|^{2} d a+(l-1)\right. \\
& \left.\times(l+2) \int_{0}^{R} \eta a^{2}\left|K_{l l}\right|^{2} d a\right\} .
\end{aligned}
$$

Above the transition temperature at which the neutron star becomes superfluid, the appropriate viscosity coefficient is

$$
\eta=2 \times 10^{18}\left(\frac{\rho}{10^{15} \mathrm{~g} \mathrm{~cm}^{-3}}\right)^{9 / 4}\left(\frac{T}{10^{9} \mathrm{~K}}\right)^{-2} \mathrm{~g} \mathrm{~cm}^{-1} \mathrm{~s}^{-1}
$$

This estimate should be valid at temperatures above, say, $10^{9} \mathrm{~K}$, i.e., for adolescent neutron stars.
In Table 1 we list the estimated timescales for the dominant $l=m=2$ and $l=m=3 r$-modes. The listed timescales were calculated at a fiducial temperature of $T=10^{9}$ $K$. From the listed data, we can deduce the critical rotation period at which the mode becomes unstable. We do this by solving for the zeros of

$$
\frac{1}{2 E} \frac{d E}{d t}=-\frac{1}{\tau_{\mathrm{gw}}}\left(\frac{1 \mathrm{~ms}}{P}\right)^{p_{\mathrm{gw}}}+\frac{1}{\tau_{\mathrm{bv}}}\left(\frac{1 \mathrm{~ms}}{P}\right)^{p_{\mathrm{bv}}}\left(\frac{T}{10^{9} \mathrm{~K}}\right)^{6}
$$

$$
+\frac{1}{\tau_{\mathrm{sv}}}\left(\frac{10^{9} \mathrm{~K}}{T}\right)^{2}
$$

at different temperatures. The resulting instability window for $l=m=2$ is shown in Figure 1. From the data in Figure 1 , we can deduce that the $r$-mode instability can potentially limit the star to remarkably slow rotation rates. For our specific stellar model, the instability is active for $\Omega / \Omega_{\mathrm{K}}>$ 0.049 , or periods shorter than $22.8 \mathrm{~ms}$ (at $T=3.7 \times 10^{9} \mathrm{~K}$ ). From this it is clear that the $r$-mode instability will dominate the evolution of a rapidly spinning neutron star.

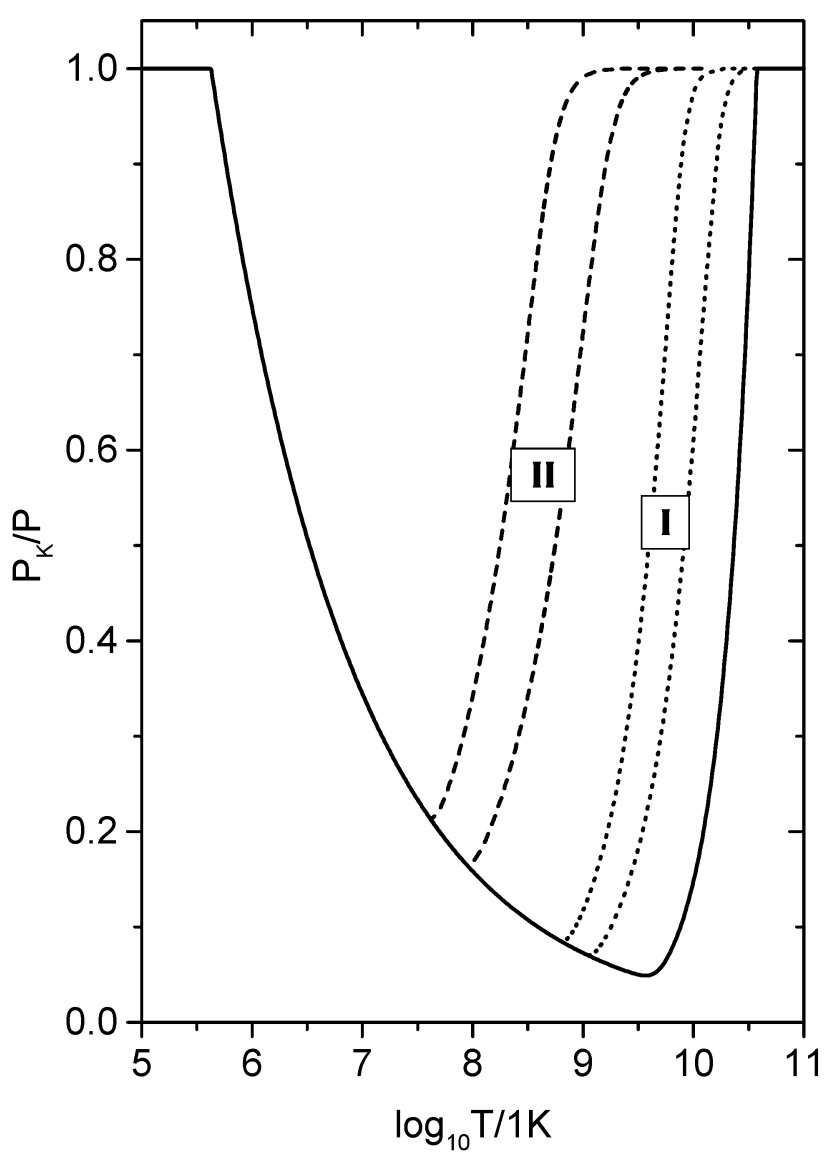

FIG. 1. - The $r$-mode instability window (for $l=m=2$ ). The mode is unstable above the solid line. At high temperatures, the growth of the instability is counteracted by bulk viscosity, while the shear viscosity prevents the instability at low temperatures. Also shown (dashed lines) are period evolutions for two different cooling scenarios: (I) standard cooling due to the modified Urca process, and (II) rapid cooling due to the direct Urca process. In both cases we assume that the star is isothermal, and we show two different evolutions, corresponding to an $r$-mode saturated at $1 \%$ and $100 \%$ of the angular momentum of the star, respectively. 
As we were at the final stage of our calculation, Lindblom et al. (1998) arrived at similar estimates using a lowest-order approach. For $l=m=2$, they get $\tau_{\mathrm{gw}}=18.91(P / 1 \mathrm{~ms})^{6} \mathrm{~s}$ and $\tau_{\mathrm{sv}}=2.52 \times 10^{8} \mathrm{~s}$, in excellent agreement with our results (see Table 1). The agreement is not so good in the case of the bulk viscosity. Lindblom et al. (1998) estimate $\tau_{\mathrm{bv}}=1.3 \times 10^{9}(P / 1 \mathrm{~ms})^{2}$ s, i.e., a factor of 70 smaller than our value. This surprisingly large difference between the two results is a consequence of the inaccurate estimate that Lindblom et al. use for the density perturbation associated with the mode.

To calculate the bulk viscosity, one needs the Lagrangian density perturbation. However, to leading order in rotation, the density perturbation is zero. Lindblom et al. (1998) work consistently only to leading order, but are able to calculate the Eulerian pressure perturbation $\delta p$ to second order in rotation directly in terms of their leading-order quantities. They use this to compute the Eulerian density perturbation to second order in rotation, from the barotropic equation of state. But the bulk viscosity depends on the Lagrangian density perturbation, which can only be calculated by fully solving the equations for the perturbation correctly to second order in rotation. We have done so in this paper, and we find that it generates a larger than expected change in the density perturbation. The Lindblom et al. (1998) approximation amounts to neglecting the term $S_{l+1 l}$ in the integrand of equation (21). It appears that the omission of this term leads to an overestimate of the bulk viscosity dissipation rate, and a low value for $\tau_{\mathrm{bv}}$. If we use the same approximation (neglecting $S_{l+1 l}$ in eq. [21]) in our calculations, we get $\tau_{\mathrm{bv}}=1.6 \times 10^{9}(P / 1 \mathrm{~ms})^{2} \mathrm{~s}$. This is in good agreement with the result of Lindblom et al., which suggests that our assertion is correct: in order to arrive at a reliable estimate for the bulk viscosity, one must include both terms in the Lagrangian variation, as we do in the present paper. That the Lagrangian variation in the density is much smaller than the Eulerian one suggests an interesting physical interpretation. In the rotating star, the fluid has moved to remain on the same (now nonspherical) surface of constant $\rho$ as in the nonrotating case. This is a property worthy of further investigation, but it is not crucial for our attempt to estimate the limit that the $r$-mode instability sets on the rotation of a young neutron star. As can be seen in Figure 1, the limiting rotation speed of the star is not a sensitive function of the size of the bulk viscosity, essentially because the bulk viscosity is such a steep function of the temperature. Therefore, the large difference between our bulk viscosity estimate and that of Lindblom et al. (1998) leads to a small effective difference in the final $\operatorname{spin}$ period $P_{f}$.

\section{ASTROPHYSICAL IMPLICATIONS}

As we have noted in the first section of this paper, there are important astrophysical implications of this instability. It limits the initial spin periods of young pulsars, it rules out the accretion-induced collapse model for the formation of the fastest millisecond pulsars, and it suggests that the formation of neutron stars could be accompanied by a strong gravitational-wave signal, lasting the cooling time.

We come back to these conclusions below, but we feel it is important first to assess the influence of various uncertainties and approximations on our results. We do not expect that the qualitative picture will change when any of the uncertainties are reduced, but the quantitative results for the limiting speed and the spin-down timescale might be affected.

One source of uncertainty is our simple stellar model. We have used a Newtonian polytrope and worked to the lowest self-consistent order in the spin. It is important that the problem be considered in the framework of general relativity. As the results of Stergioulas \& Friedman (1998) for the $f$-mode instability show, the critical rotation period (at which the mode becomes unstable) changes by something like $15 \%$ once general relativity is incorporated in the calculation. It is possible that the $r$-modes are affected in a similar way. Furthermore, a relativistic calculation is required if we want to investigate issues related to the equation of state. Within a Newtonian calculation, a comparison of results for different equations of state is not meaningful, since all realistic neutron star equations of state are for relativistic models and there is no one-to-one correspondence with the Newtonian problem. For example, for a given central density, our chosen polytrope $p=\kappa \rho^{\Gamma}$ will not produce stars with the same $M$ and $R$ in Newtonian theory and general relativity. Fully relativistic mode calculations for rotating stars are very difficult, and it may be some years before reliable results are obtained from efforts that are only now beginning. However, initial investigations within the slow-rotation approximation for relativistic stars (Hartle \& Thorne 1968) should be possible more quickly.

Another uncertainty affecting our conclusions is the initial rotation of the star after collapse. Clearly, if the initial spin of the star is small (which would be the typical case in the model of Spruit \& Phinney 1998), then the instability will not operate. In particular, there would be no gravitational radiation. But this seems to us to be unlikely to hold in all cases; the angular momentum required to make the star spin at $\Omega_{\mathrm{K}}$ is small in the initial degenerate core, and the $16 \mathrm{~ms}$ pulsar in N157B is clear evidence that some pulsars are born spinning rapidly.

Another important initial condition that we have assumed is uniform rotation, but nonaxisymmetric collapse is unlikely to leave the star in perfect rigid rotation. If the $r$-mode phase is preceded by a dynamical phase involving short-lived bar-mode instabilities (e.g., Houser et al. 1994; Rampp, Müller, \& Ruffert 1998), then the angular velocity field in the subsequent axisymmetric star in which the $r$-mode will grow may be highly differentially rotating. This may have some effect on the dynamics and growth of the instability (the $r$-modes have not been investigated in differentially rotating models), and it may also have an effect on the final period. For example, it could happen that the inner and outer regions of the star cool at different rates (see below), or that viscosity affects them differently (especially when superfluidity sets in). Then gravitational radiation may extract angular momentum from the outer layers, say, spinning them down to near $P_{f}$, but the inner core would remain rapidly rotating. Once the instability ends, the star would eventually become uniformly rotating, spinning up the outer layers. It may be that some such process was at work in N157B.

The physics of neutron stars also provides important uncertainties. Here one can hope that observations, coupled with better calculations, will provide evidence about this physics. One possible uncertainty is the cooling rate. As is clear from Figure 1, the effect of the $r$-mode instability depends largely on the temperature of the star. To assess the astrophysical role of the instability, we must assume a 
cooling scenario for a nascent neutron star. In the standard model, neutron stars are born at a temperature well above $10^{10} \mathrm{~K}$ and cool through the modified Urca process to $10^{9}$ $\mathrm{K}$ in something like $1 \mathrm{yr}$. Results obtained for this model by Nomoto \& Tsuruta (1987) are reasonably well approximated by

$$
\frac{T_{c}}{10^{9} \mathrm{~K}}=19.156\left(\frac{1 s}{t}\right)^{0.159}
$$

where $T_{c}$ is the central temperature of the star. Recently, it has been suggested that the star could cool much faster than this. The idea is that the proton fraction in the star could be sufficiently large to make the direct Urca process possible. Then the star would cool according to (Lattimer et al. 1994)

$$
\frac{T_{c}}{10^{9} \mathrm{~K}}=2.115\left(\frac{1 s}{t}\right)^{0.25}
$$

That is, the central parts of the star would cool to $10^{9} \mathrm{~K}$ in $20 \mathrm{~s}$.

When combined with the $r$-mode instability, these two cooling scenarios will inevitably lead to rather different results. To illustrate this, we have made a simple estimate of how the instability affects the rotation of the star. We do this by assuming that the mode is excited to a maximal level corresponding to a certain fraction of the total angular momentum of the star (as should be the case after an initial time of exponential growth; cf. the results of Lai \& Shapiro [1995] for the evolution of unstable ellipsoids) and that the star loses rotational energy at a rate determined by equation (26). Since we do not know at what level the mode saturates, we consider a wide range of possibilities and allow the mode to carry from $1 \%$ to all of the star's angular momentum. Results for this kind of evolutions for our two cooling scenarios are shown in Figure 1. In the case of standard cooling (case I in Fig. 1), the star reaches a final period (the $r$-mode becomes stable) in the range $13.2 \leq P / 1$ $\mathrm{ms} \leq 15.9$, while in the rapid-cooling case (case II in Fig. 1) we get $5.2 \leq P / 1 \mathrm{~ms} \leq 6.8$.

In these examples, we assumed a uniform temperature distribution in the star. This is, however, doubtful in the case of rapid cooling. When the core of the star cools rapidly, the outer $10 \%$ (say) of the star will act as a heat reservoir, and the star will not be isothermal until after something like $10 \mathrm{yr}$ (cf. Fig. 3 of Lattimer et al. 1994). In effect, the temperature of the surface layers, where the $r$-modes are mainly located, is stalled above $10^{9} \mathrm{~K}$ for the first year(s). When this effect is taken into account, the cooling rate relevant for the $r$-modes is closer to that for the standard cooling scenario. Hence, we could expect the $r$-mode evolution to be close to case I in Figure 1 even for fast cooling. This means that the instability would be active for more than $2 \mathrm{yr}$.

The details of the evolution of the instability are very sensitive to calculations of neutron-star viscosity. The largest uncertainty seems to be in the onset of superfluidity. It is typically assumed that neutron stars become superfluid at a temperature of the order of $10^{9} \mathrm{~K}$ (see Fig. 1 of Nomoto $\&$ Tsuruta 1987). As was shown by Lindblom \& Mendell (1995), the so-called mutual friction kills the $f$-mode instability completely in a superfluid star. This effect is also likely to counteract the $r$-mode instability. If superfluidity sets in first at the center of the star, it could lead to a complex spin evolution, particularly if the star is differentially rotating. An accurate study of $r$-modes in a superfluid neutron star is needed before the relevance of the instability for older, colder stars can be properly assessed (although see Andersson et al. 1998 for a discussion based on the present results).

Within these uncertainties, the simple calculations presented here and in Lindblom et al. (1998) are consistent with the limited data that we have on pulsar initial spin rates. The agreement with the presumed initial rotation period of the Crab pulsar of roughly $19 \mathrm{~ms}$ is extremely good. If other uncertainties play no role, then this would argue that superfluidity did not set in until below $10^{9} \mathrm{~K}$ in this star. The recently discovered $16 \mathrm{~ms}$ pulsar associated with the supernova remnant N157B (Marshall et al. 1998) provides a more stringent test. If we assume that the estimated age of roughly $5000 \mathrm{yr}$ is correct, and that the pulsar has a braking index (not yet determined by observations) in the range between those of the Vela and Crab pulsars $(1.4<n<2.5)$, we infer an initial period of 6-9 ms, 2 to 3 times as fast as our limit. This suggests either that more accurate physics and model calculations will show that $P_{f}$ is twice as high as our present number (in which case the Crab simply formed at a slow initial speed), or that initial conditions of formation (e.g., differential rotation, as discussed above) play a significant role in determining the final spin speed. It could also be an indication that superfluidity played an important role. If the $r$-mode instability were suppressed at $T \approx 2$ $3 \times 10^{9} \mathrm{~K}$ by superfluidity, our model would agree perfectly with the observations (cf. Fig. 1). Further observations of N157B may provide some clues. In particular, it is important to be sure that the $16 \mathrm{~ms}$ period is the true rotation period and not half the true period (i.e., that we are not seeing two nearly identical pulses per rotation period).

The present estimates clearly indicate that the $r$-mode instability makes it impossible to form the fastest millisecond pulsars (at least $P<10 \mathrm{~ms}$, if not slower) through the accretion-induced collapse of white dwarfs (see, e.g., Narayan \& Popham 1989; Bailyn \& Grindlay 1990; Nomoto \& Kondo 1991). Such a star would have to form hot, since the collapse is nearly adiabatic. Even if it were spinning rapidly at first, it would have to spin down to the kind of speed seen in young pulsars. Subsequent spin-up, i.e., by continued accretion, is necessary for periods below around $10 \mathrm{~ms}$. Hence, we suggest that all fast millisecond pulsars are "recycled."

The implications for $r$-mode gravitational radiation are exciting. The amount of energy emitted depends essentially on the initial spin of the star, but the frequency evolution depends on details of the evolution of the star that are beyond the scope of this paper. Owen et al. (1998) present a first approximation to the evolution of the star as it spins down. They show that the radiation could be detectable by advanced gravitational-wave detectors. Observations by such detectors should eventually provide new insight into the physics uncertainties we have discussed here.

This radiation would be in addition to that which might be produced in the initial collapse dynamics. If the collapsed object forms initially rotating faster than the threshold for a bar-mode instability (which is near the Kepler period), then recent studies by, for example, Houser et al. (1994) and Rampp et al. (1998) indicate that a few percent of the mass will be ejected, and that the collapsed object will lose angular momentum through the bar-mode instability. The spin will still be sufficiently fast that the $r$-mode insta- 
bility takes over, and a further phase of gravitational radiation emission occurs.

The important astrophysical implications make it urgent that progress be made to reduce the uncertainties in these calculations. Fully relativistic calculations, allowing for realistic initial conditions and realistic cooling scenarios, should lead to results that can confidently be compared with radio, X-ray, and gravitational-wave observations of neutron stars.

We thank Curt Cutler, John Friedman, Yasufumi Kojima, Lee Lindblom, and Nick Stergioulas for helpful discussions. N. A. and K. D. K. thank the AEI for generous hospitality.

\author{
Abramovici, A., et al. 1992, Science, 256, 325 \\ Andersson, N. 1998, ApJ, 502, 708 \\ Andersson, N., Kokkotas, K. D., \& Stergioulas, N. 1998, ApJ, submitted \\ (preprint astro-ph/9806089) \\ Bailyn, C. D., \& Grindlay, J. E. 1990, ApJ, 353, 159 \\ Bradaschia, C., et al. 1990, Nucl. Instrum. Methods Phys. A, 289, 518 \\ Chandrasekhar, S. 1970, Phys. Rev. Lett., 24, 611 \\ Chandrasekhar, S., \& Lebovitz, N. R. 1962, ApJ, 132, 1082 \\ Cutler, C., \& Lindblom, L. 1987, ApJ, 314, 234 \\ Friedman, J. L., \& Morsink, S. 1998, ApJ, 502, 714 \\ Friedman, J. L., Parker, L., \& Ipser, J. R. 1989, Phys. Rev. Lett., 62, 3015 \\ Friedman, J. L., \& Schutz, B. F. 1978, ApJ, 222, 281 \\ Glendenning, N. K. 1996, Compact Stars (New York: Springer) \\ Hartle, J. B., \& Thorne, K. S. 1968, ApJ, 153, 807 \\ Houser, J. L., Centrella, J. M., \& Smith, S. C. 1994, Phys. Rev. Lett., 72, \\ 1314 \\ Ipser, J. R., \& Lindblom, L. 1991, ApJ, 373, 213 \\ Lai, D., \& Shapiro, S. L. 1995, ApJ, 442, 259 \\ Lattimer, J. M., van Riper, K. A., Prakash, M., \& Prakash, M. 1994, ApJ, \\ 425,802 \\ Lindblom, L. 1995, ApJ, 438, 265 \\ Lindblom, L., \& Mendell, G. 1995, ApJ, 444, 804
}

\section{REFERENCES}

Lindblom, L., Owen, B. J., \& Morsink, S. M. 1998, Phys. Rev. Lett., 80, 4843

Marshall, F. E., Gotthelf, E. V., Zhang, W., Middleditch, J., \& Wang, Q. D. 1998, ApJ, 499, L179

Misner, C. W., Thorne, K. S., \& Wheeler, J. A. 1973, Gravitation (San Francisco: Freeman)

Narayan, R., \& Popham, R. 1989, ApJ, 346, L25

Nomoto, K., \& Kondo, Y. 1991, ApJ, 367, L19

Nomoto, K., \& Tsuruta, S. 1987, ApJ, 312, 711

Owen, B., Lindblom, L., Cutler, C., Schutz, B. F., Vecchio, A., \& Andersson, N. 1998, Phys. Rev. D, 58, 084020

Papaloizou, J., \& Pringle, J. E. 1978, MNRAS, 182, 423

Provost, J., Berthomieu, G., \& Rocca, A. 1981, A\&A, 94, 126

Rampp, M., Müller, E., \& Ruffert, M. 1998, A\&A, 332, 969

Saio, H. 1982, ApJ, 256, 717

Schutz, B. F. 1983, Lect. Appl. Math., 20, 99

Spruit, H., \& Phinney, E. S. 1998, Nature, 393, 139

Stergioulas, N., \& Friedman, J. L. 1998, ApJ, 492, 301

Thorne, K. S. 1980, Rev. Mod. Phys., 52, 299

. 1987, in 300 Years of Gravitation, ed. S. W. Hawking \& W. Israel (Cambridge: Cambridge Univ. Press) 